\title{
Non-Linear Effects in the Snoek Relaxation of Nb-O
}

\author{
E.B. Hermida and F. Povolo \\ Departamento de Física, Fac. de Ciencias Exactas y Naturales, Universidad de Buenos Aires, Pabellón I, \\ Ciudad Universitaria, 1428 Buenos Aires, Argentina \\ Unidad de Actividad Materiales, Comisión Nacional de Energía Atómica, Av. del Libertador 8250, \\ 1429 Buenos Aires, Argentina \\ Consejo Nacional de Investigaciones Científicas y Técnicas (CONICET), Buenos Aires, Argentina
}

\begin{abstract}
Internal friction peaks measured as a function of temperature or frequency have been associated to non-linear processes only after studying how the amplitude of the applied stress affects the relaxation process. Here it is demonstrated that the partial derivative of the internal friction with respect to the frequency at constant temperature is a useful tool to determine that non-linear effects are involved. This analysis applied to actual data of the Snoek relaxation in Nb-O, reveals that at high interstitial contents non-linear effects appear.
\end{abstract}

\section{INTRODUCTION}

The integral representation of the internal friction (IF) of linear viscoelastic materials is given by [1]

$$
F(\omega, T)=\frac{\alpha_{d}(\omega, T)}{2 \cosh \{\ln [\omega \tau(T)]\}}
$$

where $\omega$ is the angular frequency, $T$, the absolute temperature and $\tau$, a mean relaxation time. $\alpha_{d}$ is a function not only of the relaxation strength but also of the distribution function of the different processes involved in the damping response. Taking into account that the frequency varies slightly when $F$ is measured as a function of temperature with a torsion pendulum, it was established that $[2]$

$$
\ln \tau(T)=\arg \tanh \left[-\left.\frac{\partial \ln F}{\partial \ln \omega}\right|_{T}\right]-\ln \omega
$$

on neglecting $\partial \ln \alpha_{d} /\left.\partial \ln \omega\right|_{T}$.

Particularly, eq. (2) has been applied to the Snoek IF peak measured in stress relieved polycrystalline $\mathrm{Nb}(99.999 \%)$ doped with different concentrations of oxygen in solution $[2,3]$. The temperature dependence of $\tau$ was established when the concentration of interstitials was below 1300 at. ppm. For higher concentrations no solution of eq. (2) was found at several temperatures and no explanation about this point was given [3].

In what follows it will be demonstrated that this lack of solution is due to non-linear processes involved in the measured damping. 


\section{THEORY}

In linear viscoelasticity $F$ can be expressed not only by the integrated representation given in eq. (1) but also as [1]

$$
F(\omega, T)=\frac{1}{2} \int_{-\infty}^{\infty} \Phi_{t}(T, \tau) \frac{\mathrm{d}(\ln \tau)}{\cosh [\ln (\omega \tau)]}
$$

where $\Phi_{t}$ is the tangent distribution function.

On deriving eq. (3) it is easy to show that

$$
-\left.\frac{\partial \ln F}{\partial \ln \omega}\right|_{T}=-\left.\frac{1}{F} \frac{\partial F}{\partial \ln \omega}\right|_{T}=\frac{\int_{-\infty}^{\infty} \Phi_{t}(T, \tau) \frac{\tanh [\ln (\omega \tau)]}{\cosh [\ln (\omega \tau)]} \mathrm{d}(\ln \tau)}{\int_{-\infty}^{\infty} \Phi_{t}(T, \tau) \frac{1}{\cosh [\ln (\omega \tau)]} \mathrm{d}(\ln \tau)}
$$

Since $-1 \leq \tanh [\ln (\omega \tau)] \leq 1$, it comes straightforward that

$$
\left|\frac{\partial \ln F(T, \omega)}{\partial \ln \omega}\right|_{T} \mid \leq 1
$$

Hence, if the logarithmic derivative of the IF with respect to the frequency at a constant temperature is greater than unity, then the material must follow a non-linear viscoelastic behaviour.

Consequently, if eq. (5) is not obeyed, eq. (2) gives not a real but a complex solution which has no physical sense, except that of manifesting the presence of non-linear effects.

\section{RESULTS}

The derivative given in eqs. (4) and (5) can be determined using both the free oscillating pendulum with variable moment of inertia and the forced oscillating pendulum. Particularly the Snoek peak in $\mathrm{Nb}-\mathrm{O}$ with low interstitial content $-550,770$ and 1300 at. $\mathrm{ppm}$ - measured with the first dispositive led to derivatives which satisfied eq. (5) and, consequently, the relaxation processes involved could not be established certainly as non-linear [3, 4]. On the contrary, this result may be considered a proof of the linear characteristic of the Snoek relaxation even when it is strongly pointed out that the validity of eq. (5) is not enough to assure that the process is linear.

Analogous measurements in samples with 1600, 8300 and 13500 at. ppm of oxygen, however, did not satisfy eq. (5) in the whole temperature interval of the Snoek peak [2,3]. Because of this, no solution of eq. (2) could be determined at several temperatures as illustrated in Fig. 1 [2]. In summary, at high concentrations of interstitials the IF peaks involves non-linear effects. Thus, the relaxation time cannot be derived from eq. (2) --which assumes a linear behaviour- even at those temperatures where the logarithmic derivative satisfies eq. (5).

\section{DISCUSSION}

Weller et al. [5] measured the Snoek peak of Ta with different oxygen contents up to 5900 at. ppm and determined that the peaks were highly symmetrical and fitted them with the Gaussian distribution function of relaxation times. Similarly, they tried to fit Snoek peaks in $\mathrm{Nb}$ with oxygen contents from 1000 to 8500 at. ppm using the Gaussian or the Fuoss-Kirkwood spectra but found that "distribution functions are not able to interpret the broadening of the loss maxima in $\mathrm{Nb}-\mathrm{O}$ by oxygen interaction with an accuracy sufficient to match the quality of experimental information" [6]. In order to achieve a better fitting they proposed a physical model which considers the long range 
interartions among interstitials dissolved in bcc metals. This model allows to explain not only the displacement of the maximum but also the broadening of the Snoek peak with the concentration of defects. However, these researchers remark that "at both sides of the peak some minor but systematic deviations between the experimental data points and the theoretical curve exist, for all concentrations examined" [6].

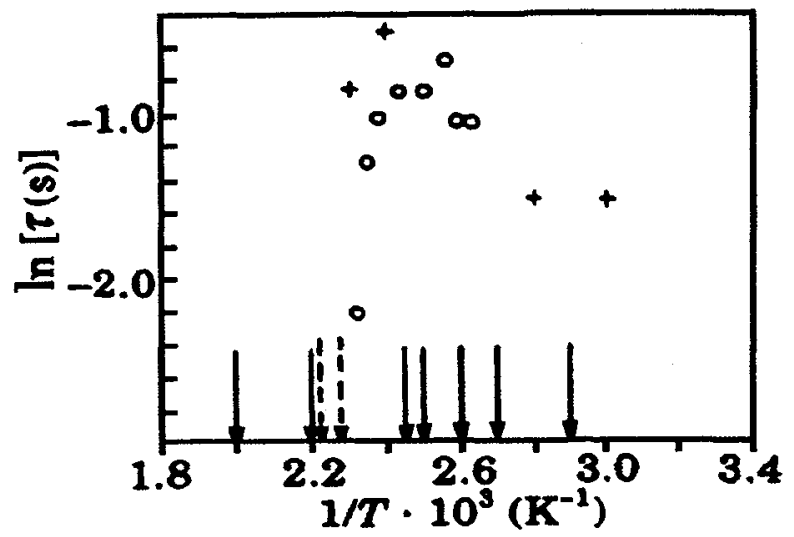

Fig. 1: $\ln \tau$ against $1 / T$ for $\mathrm{Nb}-\mathrm{O}$ samples with 1600 at. ppm (O and broken arrows) and 8300 at. ppm (+ and full arrows) of oxygen. The arrows indicate the temperatures where no solution of eq. (2) exists [2].

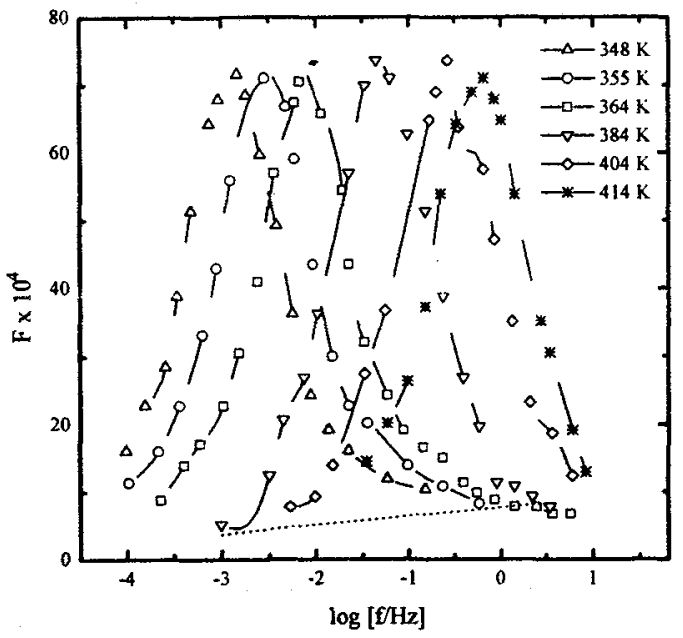

Fig. 2(a): Snoek peaks measured at the indicated temperatures

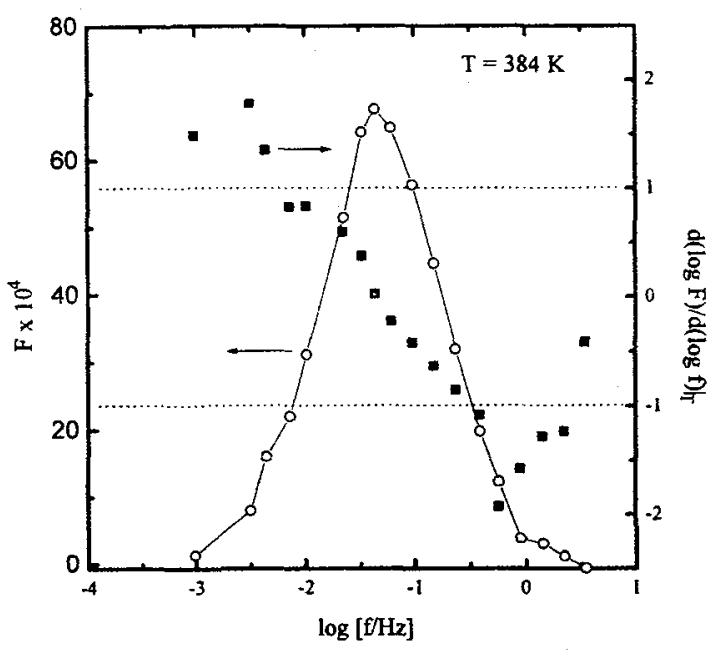

Fig. 2(b): Internal friction without the linear background indicated in Fig. 2(a) and its logarithmic derivative with respect to frequency at a constant temperature.

The non-linear characteristics determined for the Snoek relaxation at high concentrations of interstitials would explain the lack of a proper fitting found when linear viscoelasticity (distribution function or a linear physical model) is considered. Furthermore, the lack of linearity disables to employ eq. (2) to calculate relaxation times. Consequently, the parameters determined from the Arrhenius' dependence on temperature of the relaxation times calculated from eq. (2) at high oxygen 
concentrations [3] have no physical sense.

Moreover, Fig. 2(a) depicts Snoek peaks in $\mathrm{Nb}-0.15$ at.\%O samples, measured by Woirgard as a function of frequency at different temperatures [7]; they are considered also to analyze if eq. (5) is fulfilled. The logarithmic derivative of the IF peak, represented in Fig. 2(b), is calculated after subtracting a linear background - which could be determined properly only at $384 \mathrm{~K}$-.

It is noticed the importance of a precise determination of the background (even in the easiest case of being just a constant) because it can distort the calculation of the derivative of the IF peak. Effectively, if the internal friction tends to a constant different from zero at very low and very high frequeuncies, its logarithm tends to a constant and, consequently, the derivative to zero. On the contrary, if no background is present or if it is properly subtracted, the logarithm tends to $-\infty$, changing substantially the logarithmic derivative.

\section{CONCLUSIONS}

In this work it is demonstrated that the logarithmic derivative of the internal friction is an useful tool to discern whether a process is linear or not. Unfortunately, this determination is possible only if the absolute value of the derivative is greater than one. If not, that is, if eq. (5) is fulfilled, nothing can be said about the linearity without considering measurements at different amplitudes of the applied stress.

Even when the data considered here have been previously fitted using linear theories, according to our treatment it seems clear that at high concentrations of interstitials the Snoek peaks measured in $\mathrm{Nb}-\mathrm{O}$ samples manifest non-linear effects. Consequently, it will be the aim of forthcoming papers to review the existing theories and to modify them taking into account the new experimental evidence of non-linearity.

\section{Acknowledgements}

This work has been supported partially by CONICET, the University of Buenos Aires, the Antorchas Foundation and the Proyecto Multinacional de Investigación y Desarrollo en Materiales OAS-CNEA.

\section{References}

[1] F. Povolo and C. L. Matteo, Mater. Trans., JIM 33 (1992) 824 - 833.

[2] F. Povolo, B. J. Molinas and O. A. Lambri, Il Nuovo Cim. 14 D (1992) $279-286$.

[3] O. A. Lambri, Mater. Trans., JIM 35 (1994) 458 - 465.

[4] F. Povolo and O. A. Lambri, J. Alloys and Comp. 211/212 (1994) $41-44$.

[5] M. Weller, J. X. Zhang, G. Y. Li, T. S. Kê and J. Diehl, Acta Metall., 29 (1981) 1055 -1060.

[6] G. Haneczok, M. Weller and J. Diehl, phys. stat. sol. (b) 172 (1992) 557 - 572.

[7] J. Woirgard, Scripta Metall. 11 (1977) 641 - 643. 